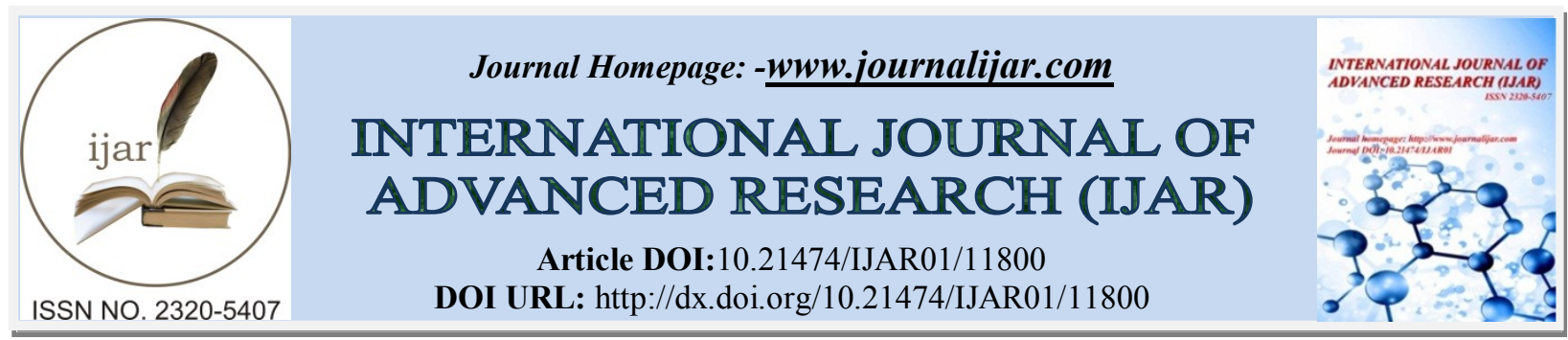

RESEARCH ARTICLE

\title{
THE NEED FOR INTERDISCIPLINARY INTERACTION BETWEEN A DENTIST AND AN INTERNIST IN TREATMENT OF SEVERE SOMATIC PATIENTS
}

Nataliya Emelyanova ${ }^{1}$, Sakal Vitalii Vitaliiovych ${ }^{2}$ and Shvets Vitalii Mykhailovych ${ }^{3}$

1. Doctor of Medical Sciences, Complex Risk Reduction of Chronic Non-Communicable Diseases; L.T. Malaya Therapy National Institute of the National Academy of Medical Sciences of Ukraine.

2. Pathomorphological Department;L.T. Malaya Therapy National Institute of the National Academy of Medical Sciences of Ukraine.

3. Candidate of Medical Sciences, Department of Reanimation and Intensive Care; L.T. Malaya Therapy National Institute of the National Academy of Medical Sciences of Ukraine.

\section{Manuscript Info}

Manuscript History

Received: 26 July 2020

Final Accepted: 31 August 2020

Published: September 2020

Key words:-

Oral Cavity, Lichen Planus, Patients

With Somatic Pathology

\section{Abstract}

This article presents a clinical analysis of the medical history of a patient being under medical treatment in the cardiovascular intensive care unit. Dental examination revealed an asymptomatic pathology of the oral mucosa in the form of a lichenoid reaction with vivid clinical symptomatology. It was concluded that these changes were of allergic origin and appeared in response to prolonged exposure to drugs. Recommendations for dental treatment of this category of patients have been substantiated.

Copy Right, IJAR, 2020,. All rights reserved.

\section{Introduction:-}

The ratio of dental and somatic diseases today is of interest among scientists of modern medicine and dentistry in particular. The relevance of studying this issue is caused by the fact that almost all diseases of internal organs are reflected to varying degrees in the oral cavity, changing the course of physiological and pathophysiological processes [1,2]. The nature of relationship between diseases of internal organs and dental lesions is multifaceted. On one side, the resulting lesions in the oral cavity depend on the presence and severity of chronic non-infectious diseases, and on the other side, it has been proven that there is negative effect of dental diseases on the risk of occurrence and aggravation of the course of the somatic pathology [3-5].There is a substantial number of studies and scientific works, where interdependence between the oral mucosa diseases, in particular periodontal tissues diseases, and changes in the respiratory system, heart and blood vessels, endocrine and digestive systems, etc., gets extensive coverage [6-9].The existing interrelationships between lesions of the organs and the oral cavity tissues and systemic pathology greatly disturbs patients, dentists and physicians, since their diagnostics involves considerable difficulties, while prevention and treatment are not always successful.

It is particularly difficult to detect dental pathology in patients that undergo treatment in the intensive care unit for life-threatening reasons, due to the fact that the life-threatening condition always gets priority, and changes in the oral cavity usually take the second place.

It is important to note that all patients with pathology of internal organs are forced to take drug therapy to maintain their somatic health for a long time, and sometimes for life [10]. There is no doubt that almost all drugs have side effects that cause certain changes in organs and tissues of the oral cavity, reducing the quality of life of patients, and 
sometimes provoking serious complications [11-14]. Today a lichenoid reaction with lichen planus (LP) is considered one of these complications.LP is quite often combined with various somatic diseases (of cardiovascular system, gastrointestinal tract, endocrine system, liver and bile ducts system) $[15,16]$. The Grinspan-Potekaev syndrome, consisting in a combination of lichen planus with diabetes mellitus and hypertension, is also widely described. There are many reasons for LP development, but today it is still not fully understood what is the trigger for occurrence of lesions in the oral cavity. However, many researchers argue that changes in the oral cavity, apart from well-known causes, can be explained from the point of the toxic-allergic theory in response to the drug load in treatment of the underlying disease $[17,18]$.

Despite the fact that LP belongs to dermatoses, in most cases having a vivid clinical picture on the skin, in $30 \%$ of cases changes can occur isolated in the oral cavity. Such lesions can cause no unpleasant subjective sensations to the patient for a long time and can be detected during a dental examination, especially with regard to patients in a serious, life-threatening condition [19-21].

Treatment of dental manifestations of LP is also a difficult task, since the use of certain drugs can be significantly limited in connection with the drug treatment of the underlying somatic pathology to prevent drug-drug substituting interaction. Below is description of a clinical case of a patient with further discussion. All pictures were taken upon obtaining the patient's consent in compliance with all ethical standards and after all appropriate documentation filling-out. At the time of examination, the patient was conscious and actively interacted.

\section{Case Presentation.}

Patient S. was in the intensive care unit with a diagnosis of dilated cardiomyopathy, pulmonary hypertension of functional class II, ventricular premature beats, ascites, hydropericardium, IIB stage cardiac distress, chronic hepatitis, type II diabetes mellitus (steroid). In 2017, the patient suffered from viral myocarditis. Long-term use of steroid drugs, highly active diuretics and antiaggregant drugs was in the history. According to the patient, he felt expressed tenderness of the lateral surface of the tongue while eating.

Objective findings: cyanotic, pale skin, submandibular, chin, external cervical and occipital lymph nodes are not palpable, no soreness in the area of their projection are observed. The tongue is mobile and not enlarged in size. There is a single defect with the diameter of $0.7-1.0 \mathrm{~cm}$, having slightly infiltrated smooth soft edges, covered with yellowish fibrinous pellicle easily removable with an instrument, on the hyperaemic base of the left lateral surface of the tongue. The defect depth is about $2.5-3 \mathrm{~mm}$. No indurations in the area of the defect are detected. Touching the defect caused tenderness and restless behavior as a result of severe soreness [Figure 1].

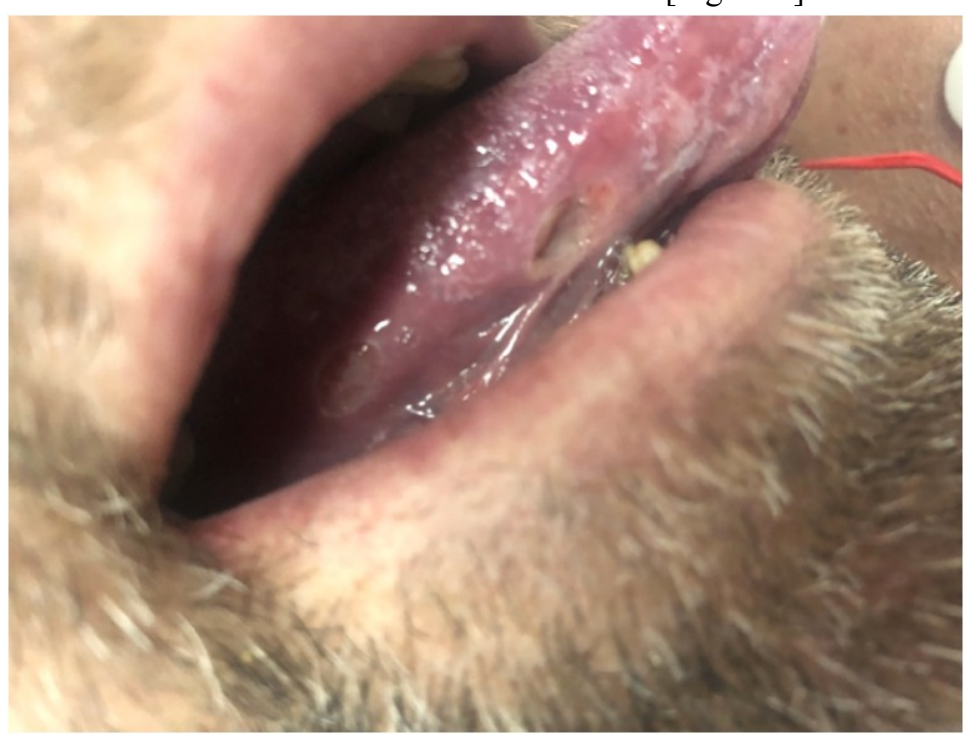

Figure 1:- Initial intraoral photos of the patient with a single defect with the diameter of $0.7-1.0 \mathrm{~cm}$ on the tongue.

In the dental arch area, it is noted that the $45^{\text {th }}$ tooth is covered with a stamped metal crown having a sharp edge close to the neck, which corresponds to the projection area of the defect on the lateral surface of the tongue. 
However, the examination revealed lesions of the oral mucosa that the patient did not complain of. The oral mucosa is edematous, white foci were determined on its surface in the area of cheeks, upper and lower lips, and in the retromolar space, rising above the surface and merging into a pattern of convoluted loops in the form of small papules. All lesions were tightly matted to the underlying tissues, failed to be removed by scraping, had bumpy surface, and caused no tenderness. The luminescent picture determined yellowish glow of the foci. These lesions were considered to be a lichenoid reaction of the oral mucosa in response to extreme exposure to the body[Figure 2].

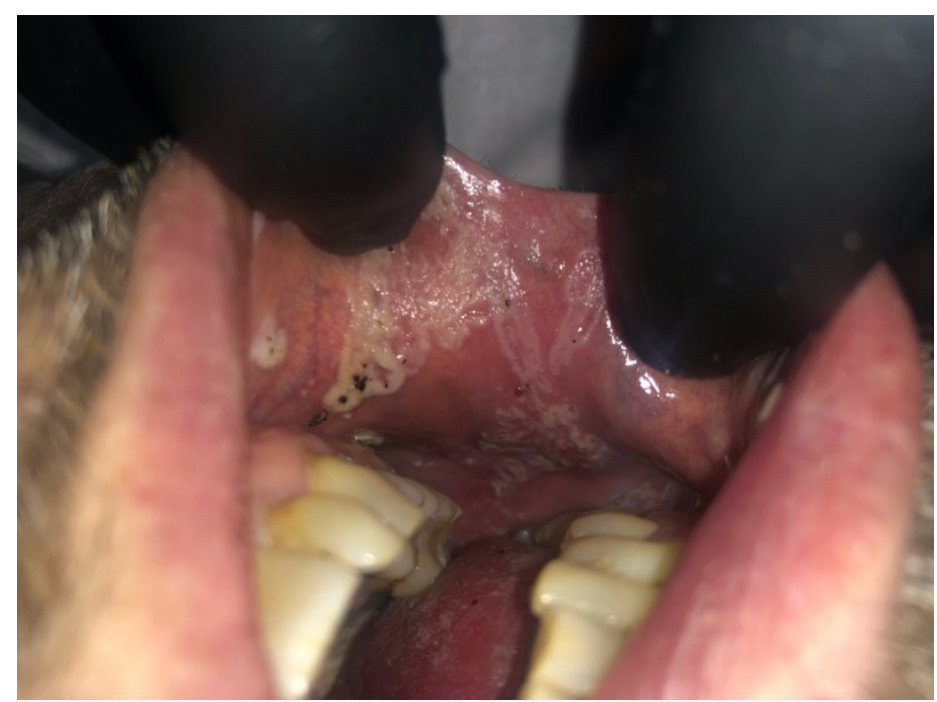

Figure 2:- Intraoral photos of the patient with a white foci were determined on its surface in the area of cheeks, upper and lower lips.

It should be noted that there were no pathological changes in the skin. The cytological study showed predominance of neutrophils (mainly at the stage of phagocytosis, lymphocytes).

In the first visit, an antiseptic treatment of the defect was carried out, the trauma factor was eliminated (sharp edges of the metal crown were grinded) in the area of the $45^{\text {th }}$ tooth, a bandage was applied to the surface of the defect (ulcer) with $0.2 \%$ sodium hyaluronate. It was recommended to applykeratoplastics 3 times a day on the ulcer area, take the lozenges with lysozyme and pyridoxine.

Repeated examinations of the patient over the next several days (after following the prescribed recommendations) allowed speaking of positive dynamics in the ulcer area on the lateral surface of the tongue -it significantly reduced in size (in diameter and depth), epithelialization and soft edges was noted, on palpationthe patient noticed significant decrease in tenderness[Figure 3].

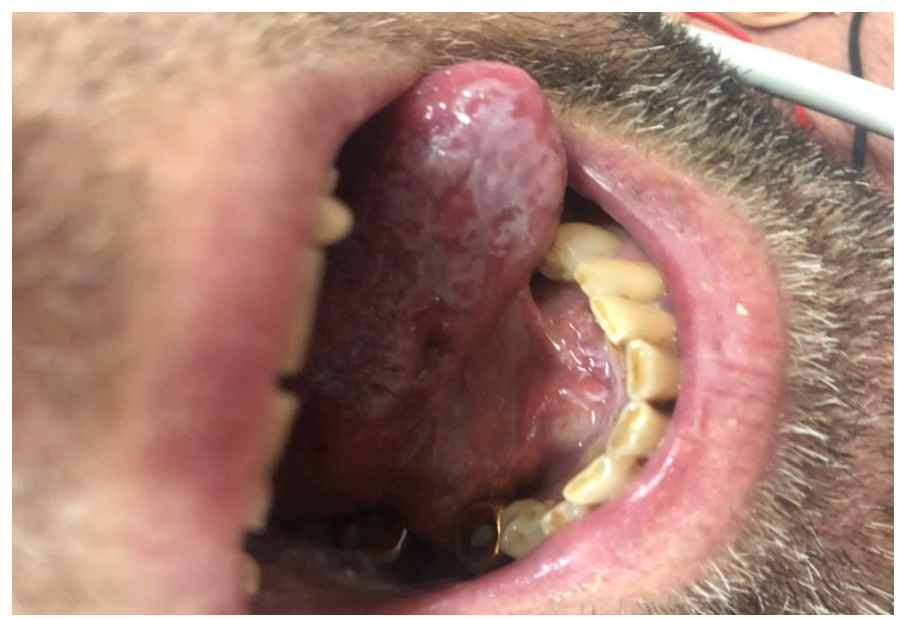

Figure 3:- Iintraoral photos of the patient with a single defect on the tongueover the next several days. 
It should also be noted that there was the significant transformation of white foci on the oral mucosa, especially in the area of the lower and upper lips, where polymorphism of the foci was noted - from white foci merged into a pattern to free covers of blisters. The Nikolsky's symptom is negative. It should be noted that the hard palate and pharynx were already involved in the process[Figure 4,5].

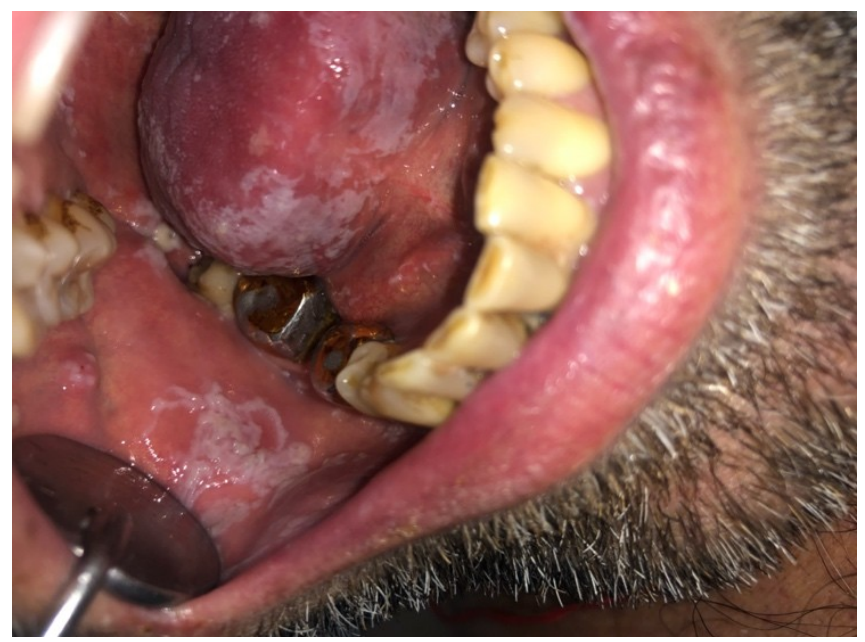

Figure 4:- Intraoral photos of the patient with a white foci were determined on its surface in the area of cheeks over the next several days.

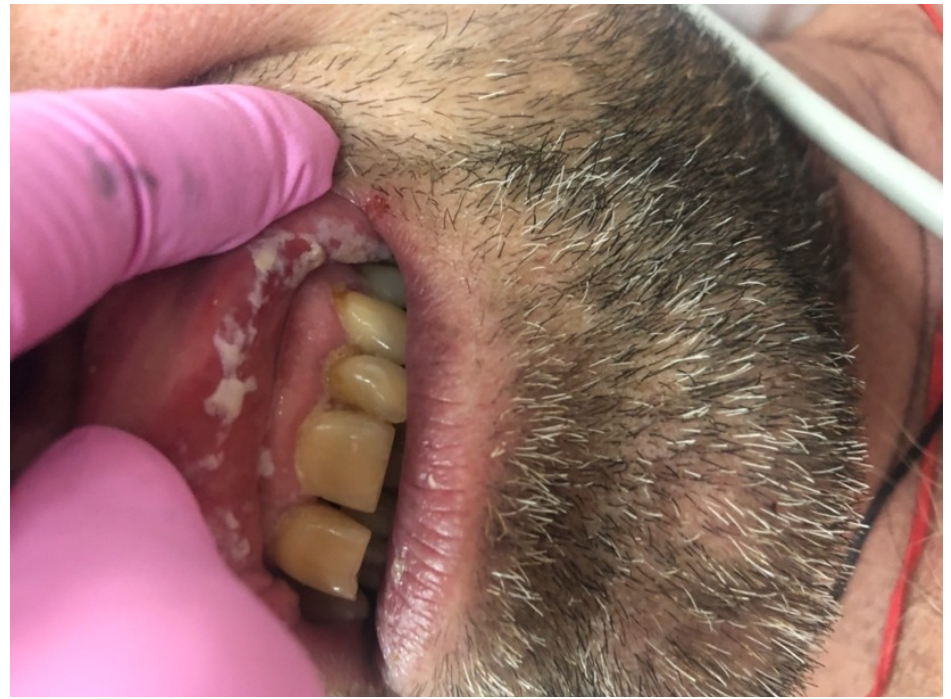

Figure 5:- Intraoral photos of the patient with a white foci in the area of the lower and upper lips, where polymorphism of the foci was noted - from white foci merged into a pattern to free covers of blisters.

For differential diagnostics of white lesions, the following was done: express swabbing for Candida albicans, PCR test for the main viruses, cytological study of polymorphic foci for the presence of cells with acantholysis symptoms. The touch smear (express method) detected cells of stratified squamous epithelium, infiltration (leukocytes up to $1 / 2$ in the field of view) and spores (not mycelium) of Candida fungi (which cannot be a criterion for fungal infection).PCR test data: hepatitis B virus (HBV), HBsAg and hepatitis $\mathrm{C}$ virus (HCV), total IgA $+\mathrm{G}$ antibodies were negative. The tests for HIV-1/2 type (IG G, IgM antibodies) and CMV were also negative. However, signs of secondary immunodeficiency were determined, particularly the positive result for EBV (EpsteinBarr virus) and HSV-1,2 (herpes simplex virus types 1 and 2). No acantholytic cells were found during cytological examination. The out-of-tolerance blood test parameters were the significant leukocytosis (WBC-20.2*10^9/L), the excess of granulocytes (GRA-17.9*10^9/L). The blood glucose level was $11.2 \mathrm{mmol} / \mathrm{l}$. 
The patient received no further treatment for white lesions due to the fact that he was transferred to another clinic by place of residence, however, recommendations were transferred to the dentist who would provide assistance (rationale for the recommendations are given in the section "Discussion"). Besides, the patient was recommended to consult an infection disease doctor on the results of PCR test of the oral cavity.

\section{Discussion:-}

The lesions are present only on the oral mucosa and manifestations of the following "white" foci are absent: leukoplakia (no lace pattern of lesions, the nature of luminescence during luminescent diagnostics), pseudomembranous candidiasis (foci tightly mate to the surface, stay unremoved by scraping, no pseudomycelium in the smear), pemphigus (the Nikolsky's symptom is negative, no acantholytic cells in cytology), erythema multiforme exudative (clinical data).

The factor worth noticing was the absence of tenderness and discomfort in the area of the focal lesions, which, in our opinion, may be associated with violation of trophic processes of a generalized nature as a result of the main somatic pathology, particularly, vascular patency violation, generation of hypoxia phenomena, oxidative stress and energy deficiency in tissues. All lesions were characterized as anallergic-genesis lichenoid reaction of the oral mucosa in response to extreme exposure to drugs, manifested in a combination of lichen planus forms (typical, exudative hyperemic and bullous).

It is well-known that the main treatment of lichenoid manifestations of allergic genesis is elimination of the etiological factor, local or systemic therapy and recommendations for oral care. The whole difficulty consists in the fact that that patients with a somatic pathology have to maintain their health with the help of specially selected medicines that are not always possible to cancel or replace with any others. Also, as noted earlier, the patient had type 2 diabetes mellitus (steroidal), which excluded the use of systemic corticosteroids or injections under the lesions for their reduction. Applying corticosteroid ointments on the oral mucosa also has its limitations, despite their low concentration during absorption. Therefore, for further management of this patient, recommendations were given both for the dentist and the patient: short-term applications of ointments or gels containing corticosteroids and keratoplastics to the areas of the oral mucosa; use of keratolytics (removal of hyperkeratosis spots); applying dressings on the oral mucosa with local immunocorrective drugs; professional oral hygiene. The program of individual hygienic care for the oral cavity was developed for the patient with special products and hygiene items selected.

\section{Conclusions:-}

1. Interdependence of somatic and dental pathologies reflects their comorbidity.

2. Regular dental examinations of patients with somatic pathology in the acute period, including those in intensive care units, provide the necessary control over the state of the oral cavity, help to reveal emerging disorders and are an integral part of highly specialized dental care.

Thus, development of interdisciplinary interaction between dentists and internists is aimed at developing unified approaches to treatment of patients with somatic and dental diseases.

\section{Authors' contributions:}

According to the order of the Authorship

\section{Declaration of patient consent}

The authors certify that they have obtained all appropriate patient consent forms. In the form the patient(s) has/have given his/her/their consent for his/her/their images and other clinical information to be reported in the journal. The patients understand that their names and initials will not be published and due efforts will be made to conceal their identity, but anonymity cannot be guaranteed.

\section{Financial support and sponsorship:}

Nil. 


\section{Conflicts of interest:}

There are no conflicts of interest.

\section{References:-}

1. Chapple IL, Genco R. Working group 2 of joint EFPAAP. Diabetes and periodontal diseases: Consensus report of the Joint EFP/AAP Workshop on Periodontitis and Systemic Diseases. Journal of Clinical Periodontology 2013; 40(Suppl 14): 106-112. Available at: https://doi.org/10.1111/jcpe.12077

2. Boillot A, Range H, Danchin N, Kotti S, Cosler G, Czernichow S, Meilhac O, Puymirat E, Zeller M, Tchetche D, Bouchard P, Simon T. Periodontopathogens antibodies and major adverse events following an acute myocardial infarction: Results from the French Registry of Acute ST-Elevation and Non-ST-Elevation Myocardial Infarction (FAST-MI). Journal of Epidemiology and Community Health 2016; 70: 1236-1241. Available at: https://doi.org/10.1136/jech-2015-207043

3. D’Aiuto F, Gkranias N, Bhowruth D, Khan T, Orlandi M, Suvan J, Masi S, Tsakos G, Hurel S, Hingorani AD, Donos N, Deanfield JE, Group T. Systemic effects of periodontitis treatment in patients with type 2 diabetes: A 12-month, singlecentre, investigator-masked, randomised trial. Lancet Diabetes and Endocrinology. 2018; 6: 954-965. Available at: https://doi.org/10.1016/S2213-8587(18)30038-X

4. Kaushal S, Singh AK, Lal N, Das SK, Mahdi AA. Effect of periodontal therapy on disease activity in patients of rheumatoid arthritis with chronic periodontitis. Journal of Oral Biology and Craniofacial Research2019; 9: 128-132. Available at: https://doi.org/10.1016/j.jobcr.2019.02.002

5. EmelyanovaNataliyaYu,EmelyanovDmitryV. Chronic Periodontitis as a Factor of Initial and Secondary Systemic Low Intensity.Saudi Journal of Oral and Dental Research 2020:5(7); 306-309. DOI: 10.36348/sjodr.2020.v05i07.001

6. Rita Del Pinto, Davide Pietropaoli, Eva Munoz-Aguilera, Francesco D’Aiuto, Marta CzesnikiewiczGuzik, Annalisa Monaco, Tomasz J. Guzik, Claudio Ferri, Periodontitis and Hypertension: Is the Association Causal? High Blood Pressure \& Cardiovascular Prevention 2020. Available at: http:// 10.1007/s40292-02000392-z

7. EmelyanovaNYu, GalchinskayaVY, BondarTN.Features of the metabolism of nitric oxide in periodontal disease in patients with COPD in combination with CHD. World of Medicine and Biology2018; 1(63):2831.DOI: 10.26724/2079-8334-2018-1-63-28-31

8. EmelyanovaNYu. Features of differentiation of gingival epitheliocytes in patients with chronic obstructive pulmonary disease in combination with coronary heart disease. Word science 2017;4.(6): 20-22

9. DV Emelyanov, AA Ponomarev, VK Leontiev.Cytomorphometric indicators of the gingiva epitheliocytes of patients with ischemic heart disease on the background of taking acetylsalicylic acid. Drug Invention Today2019; 12(10): 2333-2335.Available at: http:// jprsolutions.info ISSN:0975-7619

10. IsayevaG, RieznikL, BuriakovskaO, Vovchenko M, EmelyanovaN, Shalimova A.The impact of group and individual training on hemodynamics, lipid metabolism, physical activity and quality of life in patients with high and very high cardiovascular risk. Wiadomosci lekarskie 2019; LXXII (12): 2315-2323. Available at: http:// journal-articleEID: 2-s2.0-85080993276

11. EmelyanovaNYu. Effect of basic therapy of chronic obstructive pulmonary disease in combination with coronary heart disease on physical parameters of the oral fluid. Sciences of Europe2017;1 (20):56-59.

12. Bakhtiari S, Sehatpour M, Mortazavi H, Bakhshi M. Orofacial manifestations of adverse drug reactions: a review study. Clujul Med. 2018; 91(1):27-36.

13. VV. Boras, A Andabak-Rogulj, VBrailo. Adverse drug reactions in the oral cavity. Acta Clin Croat. 2015; 54: 208-215.

14. Sag C, Ozden FO, Acikgoz G, Anlar FY. The effects of combination treatment with a long-acting beta2-agonist and a corticosteroid on salivary flow rate, secretory immunoglobulin A, and oral health in children and adolescents with moderate asthma: a 1-month, single-blind clinical study. Clin Ther. 2007; 29(10):2236-2242.

15. Anisimova I, Simonyan L. Thefrequency of combination of oral lichen planus with somatic pathology and local unfavorable factors of the oral cavity. Actual problems in dentistry 2019. 15: 16-22. Available at: http:// 10.18481/2077-7566-2019-15-1-16-22.

16. Gupta Sonia,JawandaManveen. Oral Lichen Planus: An Update on Etiology, Pathogenesis, Clinical Presentation, Diagnosis and Management. Indian Journal of Dermatology 2015; 60:222. Available at: http:// 10.4103/0019-5154.156315.

17. Ana Pejcic. Drug-Induced Oral Reactions, Emerging Trends in Oral Health Sciences and Dentistry, Mandeep Singh Virdi, IntechOpen 2015 DOI: Available from: https://www.intechopen.com/books/emerging-trends-inoral-health-sciences-and-dentistry/drug-induced-oral-reactions 
18. KamathVV, SetlurK, Yerlagudda,K. Oral lichenoid lesions - a review and update. Indian journal of dermatology 2015; 60(1): 102. Available at: // https://doi.org/10.4103/0019-5154.147830

19. Mathur M, Acharya P, Karki A, Kc N, Shah J, Jha A. Isolated lichen planus of lip: Diagnosis and treatment monitoring using dermoscopy. Clin Case Rep. 2018;7(1):146-148. Published 2018 Nov 22. doi: $10.1002 / \mathrm{ccr} 3.1933$

20. Shavit E, Hagen K, Shear N. Oral lichen planus: a novel staging and algorithmic approach and all that is essential to know. F1000research. 2020;9. DOI: 10.12688/f1000research.18713.1.

21. Lauritano D, Arrica M, Lucchese A. Oral lichen planus clinical characteristics in Italian patients: a retrospective analysis. Head Face Med. 2016;12:128. 10.1186/s13005-016-0115-z. 